\title{
Comparison of Awareness of Patient Parameters between Two Groups of Caregivers in Intensive Care Unit
}

\author{
Mohandeep Kaur, Saurav Mitra Mustafi, Manila Singh, Anupama Gill Sharma, Vinod Bala Dhir, Jyoti Sharma \\ Department of Anesthesia and Intensive Care, PGIMER, Dr. RML Hospital, New Delhi, India
}

\section{Abstract}

\begin{abstract}
Aim of the Study: The overlap in the scope of duties performed by two core groups of Intensive Care Unit caregivers, the doctors and nurses may lead to gaps in awareness of patient-related parameters among them. Our study tested the hypothesis that there is no difference in the awareness of patient-related parameters between the two study groups (doctors and nurses). Materials and Methods: A questionnaire-based study, incorporating various aspects of a patient's medical care was designed. Pro forma for 100 patients was filled by doctors and nurses divided into two groups of 100 each (50 junior residents [JRs] and 50 senior residents [SRs] in the doctors' group). Statistical analysis of categorical data was done by Chi-squared test and interval data by $t$-test. A subgroup analysis was done for comparison between nurses SRs and JRs as independent groups. $P<0.05$ was considered statistically significant. Results: There was no statistically significant difference between the two groups (doctors and nurses) in terms of percentage of correct responses in the questionnaire $(P=0.655)$. A highly significant difference between the knowledge of SRs and nurses was found with a $P=0.0001 . P<0.0001$ was calculated for the SRs versus JRs which was highly significant. Conclusions: As a group, doctors (SRs and JRs) did not reflect any difference in awareness of patient-related parameters when compared to nurses. However, SRs were more knowledgeable about the patient-related parameters when compared independently with the JRs and the nurses.
\end{abstract}

Keywords: Doctors, junior residents, nurses, patient parameters, senior residents

\section{INTRODUCTION}

Doctors and nurses form the core caregivers of Intensive Care Units (ICUs). The nurses are mostly involved in patient care such as postural changes and oral care while the doctors are involved in patient management such as pharmacological prescription, optimizing hemodynamics, and physiology of the patient. There are though some grey areas where the responsibilities may overlap and ICU academics are usually structured to impart equivalent training to these core ICU teams in basic patient management. We, therefore, planned our study to compare the awareness of patient parameters between the two groups of caregivers in ICU.

\section{Materials and Methods}

After obtaining ethical clearance, we conducted a questionnaire-based study over a $6 \frac{1}{2}$ months duration in multispecialty ICUs of our tertiary care teaching hospital. Our team used a standalone $a d$ hoc questionnaire to scrutinize the possibility of difference in awareness of basic patient

\begin{tabular}{|l|l|}
\hline \multicolumn{2}{|c|}{ Access this article online } \\
\hline Quick Response Code: & Website: \\
\hline & www.ijccm.org \\
\hline & \\
\hline
\end{tabular}

parameters among staff members in with differing clinical roles in our ICU. The questions were tailored to include the specific needs and structure of our ICU. As a general practice, FAST HUGS BID and FASTHUGS-MAIDENS are used in the ICU during the grand rounds to provide an all-inclusive analysis of patient condition on the day. Most of the questions directly test the parameters included in the sign-out, but additional points like posture change were included, done solely by nurses. As an institutional practice, we nurse all our patients in a slight head up position unless (and very rarely) contraindicated and was henceforth excluded from the questionnaire. Our questionnaire was designed to not only test the daily patient parameter awareness but also review the awareness of the patient

Address for correspondence: Dr. Mohandeep Kaur, Consultant, Department of Anesthesia and Intensive Care, PGIMER, Dr. RML Hospital, New Delhi - 110 001, India. E-mail: mdkaur@gmail.com

This is an open access article distributed under the terms of the Creative Commons Attribution-NonCommercial-ShareAlike 3.0 License, which allows others to remix, tweak, and build upon the work non-commercially, as long as the author is credited and the new creations are licensed under the identical terms.

For reprints contact: reprints@medknow.com

How to cite this article: Kaur M, Mustafi SM, Singh M, Sharma AG, Dhir VB, Sharma J. Comparison of awareness of patient parameters between two groups of caregivers in intensive care unit. Indian J Crit Care Med 2017;21:665-70. 
history and underlying pathology and major comorbidities. Our study was not designed to test the medical knowledge of the clinical staff but was framed to review if the staff with the assigned clinical roles were aware of the basic parameters which we believe might change the clinical outcome of the patients. Informed consent for confidentiality was taken from the doctors and nurses enrolled in the study before enrollment.

The patients in our ICU are routinely looked after by a team of certified Anaesthesiology senior residents (SRs) rotated 2 monthly as a team of four, led by a senior faculty member along with junior residents (JRs) (also rotated as a team of four) who are nonspecialized trainee doctors who have attained their M.B.B.S. degree. The staff nurses do the duties in four shifts. The posting of the nurses is not changed from one ICU in our institution till need arises to relocate them under special conditions. The nurse to patient ratio is at least 1:2 at all times. The nurses have their grand rounds at 8 am and the doctors at $9 \mathrm{am}$. The over is given in written in separate registers for the nurses and doctors. The scoring system used by all team members in our ICU is Sequential Organ Failure Assessment (SOFA) score. ${ }^{[1-3]}$ European Pressure Ulcer Advisory Panel National Pressure Ulcer Advisory Panel and Pan Pacific Pressure Injury Alliance consensus document (2014) is used for pressure sore classification. ${ }^{[4]}$

The principal investigator who had complete knowledge of the patient-related information did the analysis of the pro forma filled up by the doctors and nurses in separate rooms on randomly selected days of the month, randomization done through computer-generated random numbers. The patients' whose information was gathered were similarly randomly selected, randomization being done among patients admitted for 3 days or more on any given day. Pro forma were handed over to the medical staff at 11 am of selected days without their prior knowledge, assuming completion of both nursing and physician grand rounds. No patient was included more than once in the study. Questionnaire for 100 patients was filled by doctors and nurses divided into two groups of 100 each. 50 pro forma were filled by JRs, 50 by SRs and 50 more by nurses in the ICU. Pro forma for one patient was filled by one doctor (either JR or SR) and one nurse at a time. We thus collected data in the form of 200 pro forma. Each doctor (SRs or JRs) filled up pro forma for not more than two patients, and each nurse had filled up pro forma for not $>3$ patients.

Categorical data were analyzed by Chi-square test and interval data by Mann-Whitney test for independent data due to the overall and subgroup data being positively skewed as per Shapiro-Wilks test SPSS statistical package (version 17.0; SPSS Inc., Chicago, IL, USA). An alpha criterion of 0.05 was selected. $P<0.05$ was considered statistically significant. A post hoc power analysis was done for groups in which a significant difference was seen.

\section{Questionnaire}

1. Do you know why the patient is in ICU?

2. Is there any other chronic history or comorbidity?

3. Do you know of the no. of organ failures in the patient?
4. Is the antibiotic therapy as per culture sensitivity or empirical?

5. Has any special investigation apart from routine been sent?

6. Do you know the total lymphocyte count (TLC), differential leukocyte count (DLC) trend of the day?

7. Is the electrolyte status deranged?

8. Is there any or new organ failure today?

9. Is the patient's course towards better or worse?

10. Is there any redness or ulcer in oropharynx or pressure points?

11. How often is posture changed in ICU?

12. Is there any vaginal discharge present (in case of female patients)?

13. Is there any plan to change central line/endotracheal tube/ tracheostomy/Foleys today?

14. Is there any significant output from drain/Ryle's tube?

15. Is central line present?

16. Is suction being done regularly, how thick are the secretions?

17. Was weaning trial done today?

18. Whether patient's ABG is acceptable?

19. Whether the patient is on any inotropes or any other infusion?

20. Whether patient is on enteral or parenteral feed or a combination of both?

21. Any critical event for the patient in ICU?

22. Is patient receiving deep vein thrombosis (DVT) prophylaxis?

23. Has informed consent for treatment been taken?

24. Is Physiotherapy being done regularly and how many times/day?

25. Has informed consent for patient's prognosis been taken?

\section{RESULTS}

A Mann-Whitney analysis of the two test groups, doctors and nurses was done after tabulating the percentage of correct responses in each group for all the questions. The $P=0.6551$ which was statistically not significant [Figure 1]. A subgroup analysis was also done for nurses versus JRs, nurses versus SRs, and JRs versus SRs. A $P=0.0665$ was achieved for the comparative analysis of the knowledge of nurses versus JRs [Figure 2], prompting us to deduce an absence of any significant difference between the two groups. A highly significant difference between the knowledge of SRs and nurses was found with a $P=0.0001$ [Figure 3]. A $P<0.0001$ was calculated for the SRs versus JRs suggesting a highly significant difference of knowledge levels between the two groups [Figure 4].

Individual Chi-squared analysis for the difference between the three subgroups for each question was also done based on correct and wrong responses [Table 1].

\section{DISCUSSION}

For ICU caregivers, continued accession of knowledge, skills and inter-professional communication is essential for 


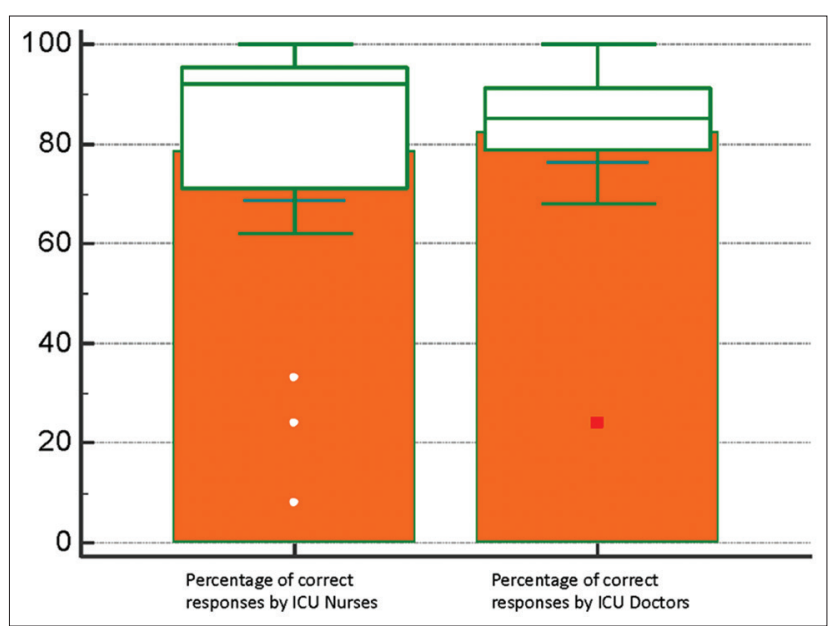

Figure 1: As seen in the graph, for a 95\% confidence interval to detect a difference between the means was not present for the doctors group in comparison with the nurses group

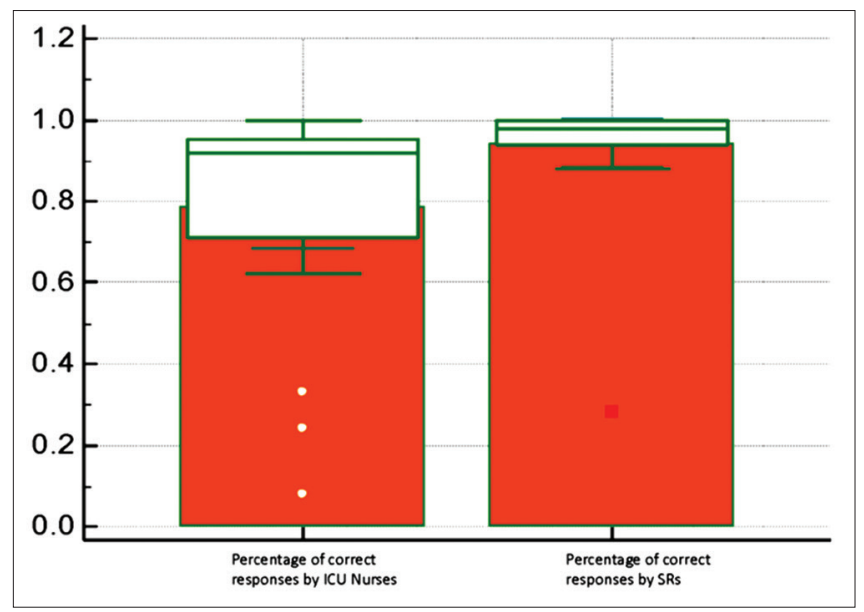

Figure 3: As seen in the graph, for a 95\% confidence interval to detect a difference between the means was present for the senior residents group in comparison with the nurses group

imparting quality care. Many studies have tried to evaluate the impact of nursing care in managing ICU patients in areas such as weaning from mechanical ventilation, chronic pain and glycemic control, areas earlier dealt with solely by doctors. ${ }^{[5-8]}$ Gaps in awareness of patient parameters in the core team might hinder patient care. Time-to-time audits conducted in the ICU are good tools to find out these gaps. Hence, our questionnaire included questions pertaining to patient history, treatment, current status, investigations, prognosis, general care, nutrition, etc., which are paramount in laying out quality improvement strategies for our ICU. ${ }^{[9]}$

The primary aim of our study was to compare awareness of patient parameters amongst the nurses and doctors. The audit was also required for evaluation of the awareness of patient parameters amongst both SRs and JRs as the JRs participate in ICU care of patients alongside the SRs. There was the absence of any significant difference between the awareness of both the groups. This prompted us to do a subgroup analysis of

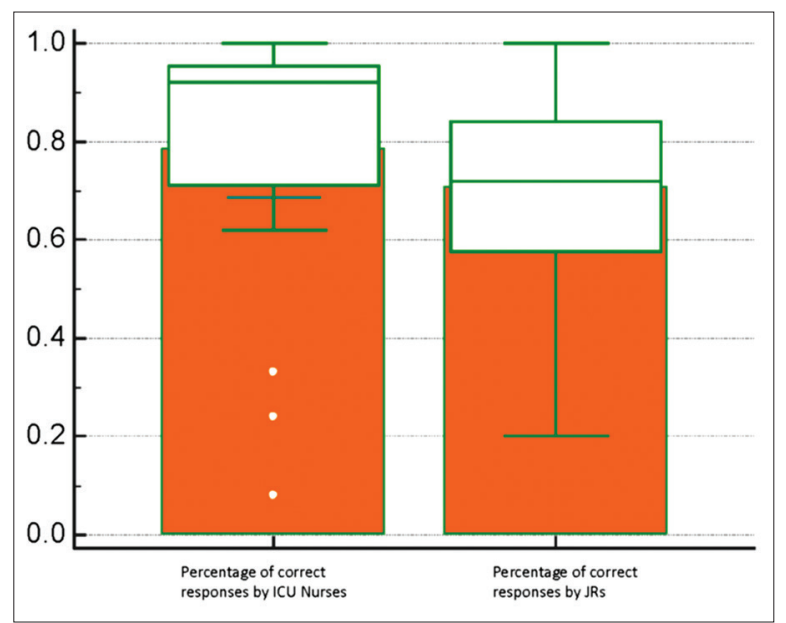

Figure 2: As seen in the graph, for a 95\% confidence interval to detect a difference between the means was not present for the nurses group in comparison with the junior residents group

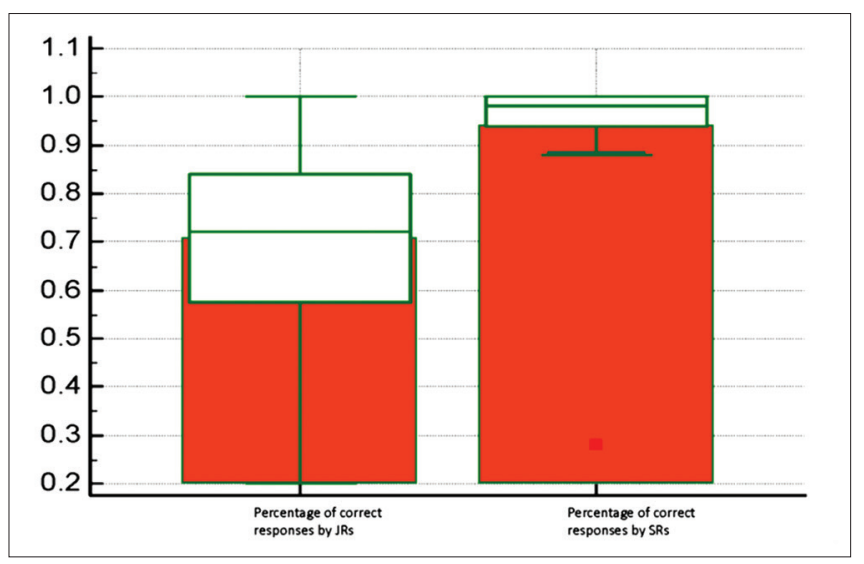

Figure 4: As seen in the graph, for a 95\% confidence interval to detect a difference between the means was present for the senior residents group in comparison with the junior residents group

the nurses, SRs and JRs as three different subgroups, as there was a large perceived difference in the knowledge of the SRs as compared to the JRs while interpreting the significance of the results with the SRs and JRs clubbed in one large group. We detected a highly significant difference of the knowledge between the SRs as compared to the other two groups $(P<0.001)$. The knowledge of nurses though was not statistically significant compared to JRs $(P=0.07)$. A post hoc power analysis was also done for comparison between the subgroups of nurses, JRs and SRs. Assuming the $\alpha$ criterion to be 0.05 , with a $95 \%$ confidence interval, a $99.9 \%$ power was achieved to reject the null hypothesis between the groups of JRs versus SRs and the groups of nurses versus SRs.

The decision to admit a patient in ICU is based on several parameters which vary across institutions with respect to diagnosis, priority, and triage. ${ }^{[10]}$ Questions 1-3 were about the presentation and history of the patients. SRs were more aware than the nurses or the JRs in these areas. Nurses were significantly more aware of the patients' comorbidities than 


\begin{tabular}{|c|c|c|c|c|c|c|c|c|c|c|c|c|c|c|c|}
\hline Questions & 1 & 2 & 3 & 4 & $P$ & 1 & 2 & 5 & 6 & $P$ & 3 & 4 & 5 & 6 & $P$ \\
\hline 1 & 71 & 29 & 36 & 14 & 0.8984 & 71 & 29 & 50 & 0 & $<0.0001$ & 36 & 14 & 50 & 0 & $<0.0001$ \\
\hline 2 & 67 & 33 & 43 & 7 & 0.0131 & 67 & 33 & 50 & 0 & $<0.0001$ & 43 & 7 & 50 & 0 & 0.0061 \\
\hline 3 & 79 & 21 & 20 & 30 & $<0.0001$ & 79 & 21 & 49 & 1 & 0.0019 & 20 & 30 & 49 & 1 & $<0.0001$ \\
\hline 4 & 75 & 25 & 35 & 15 & 0.5139 & 75 & 25 & 46 & 4 & 0.0129 & 35 & 15 & 46 & 4 & 0.0050 \\
\hline 5 & 71 & 29 & 40 & 10 & 0.2362 & 71 & 29 & 50 & 0 & 0.0004 & 40 & 10 & 50 & 0 & 0.0138 \\
\hline 6 & 8 & 92 & 23 & 27 & $<0.0001$ & 8 & 92 & 49 & 1 & $<0.0001$ & 23 & 27 & 49 & 1 & $<0.0001$ \\
\hline 7 & 62 & 38 & 44 & 6 & 0.0010 & 62 & 38 & 50 & 0 & $<0.0001$ & 44 & 6 & 50 & 0 & 0.0115 \\
\hline 8 & 92 & 8 & 40 & 10 & 0.0330 & 92 & 8 & 48 & 2 & 0.3545 & 40 & 10 & 48 & 2 & 0.0138 \\
\hline 9 & 83 & 17 & 35 & 15 & 0.0669 & 83 & 17 & 47 & 2 & 0.0617 & 35 & 15 & 47 & 2 & 0.0018 \\
\hline 10 & 96 & 4 & 41 & 9 & 0.0041 & 96 & 4 & 44 & 6 & 0.0641 & 41 & 9 & 44 & 6 & 0.4008 \\
\hline 11 & 100 & 0 & 21 & 29 & $<0.0001$ & 100 & 0 & 47 & 3 & 0.0133 & 21 & 29 & 47 & 3 & $<0.0001$ \\
\hline 12 & 24 & 12 & 10 & 6 & 0.7707 & 24 & 12 & 14 & 6 & 0.7980 & 10 & 6 & 14 & 6 & 0.6353 \\
\hline 13 & 96 & 4 & 28 & 22 & $<0.0001$ & 96 & 4 & 50 & 0 & 0.1517 & 28 & 22 & 50 & 0 & $<0.0001$ \\
\hline 14 & 95 & 5 & 36 & 14 & $<0.0001$ & 95 & 5 & 49 & 1 & 0.3768 & 36 & 14 & 49 & 1 & 0.0003 \\
\hline 15 & 96 & 4 & 42 & 8 & 0.0107 & 96 & 4 & 49 & 1 & 0.5201 & 42 & 8 & 49 & 1 & 0.0144 \\
\hline 16 & 92 & 8 & 48 & 2 & 0.3545 & 92 & 8 & 46 & 4 & 1.0000 & 48 & 2 & 46 & 4 & 0.3997 \\
\hline 17 & 71 & 29 & 40 & 10 & 0.2362 & 71 & 29 & 46 & 4 & 0.0034 & 40 & 10 & 46 & 4 & 0.0838 \\
\hline 18 & 33 & 67 & 35 & 15 & $<0.0001$ & 33 & 67 & 47 & 3 & $<0.0001$ & 35 & 15 & 47 & 3 & 0.0018 \\
\hline 19 & 92 & 8 & 35 & 15 & 0.0004 & 92 & 8 & 50 & 0 & 0.0398 & 35 & 15 & 50 & 0 & $<0.0001$ \\
\hline 20 & 91 & 9 & 29 & 21 & $<0.0001$ & 91 & 9 & 50 & 0 & 0.0287 & 29 & 21 & 50 & 0 & $<0.0001$ \\
\hline 21 & 92 & 8 & 41 & 9 & 0.0686 & 92 & 8 & 49 & 1 & 0.1447 & 41 & 9 & 49 & 1 & 0.0077 \\
\hline 22 & 92 & 8 & 20 & 30 & $<0.0001$ & 92 & 8 & 48 & 2 & 0.3545 & 20 & 30 & 48 & 2 & $<0.0001$ \\
\hline 23 & 92 & 8 & 50 & 0 & 1 & 92 & 8 & 50 & 0 & 1 & 50 & 0 & 50 & 0 & 1 \\
\hline 24 & 96 & 4 & 42 & 8 & 0.0107 & 96 & 4 & 50 & 0 & 0.1517 & 42 & 8 & 50 & 0 & 0.0032 \\
\hline 25 & 100 & 0 & 50 & 0 & 1.0000 & 100 & 0 & 50 & 0 & 1.0000 & 50 & 0 & 50 & 0 & 1.0000 \\
\hline
\end{tabular}

1: Correct answers by nurses; 4: Wrong answers by JRs; 2: Wrong answers by nurses; 5 : Correct answers by SRs; 3: Correct answers by JRs; 6: Wrong answers by SRs. JRs: Junior residents; SRs: Senior residents

the JRs. Deficient knowledge of the nurses and JRs in the indication for admission reflected in a deficiency in patients' antibiotic therapy-related knowledge (Q no. 4). As compared to the doctors on rotation in the ICUs, the nurses, in compliance with our hospital policy, are a relatively stationary population. Nurses review the medication charts every day and thus are in a perfect spot to guide antimicrobial therapy at all times without being directly involved in the prescription by monitoring decision-making and minimizing errors in prescription and compliance. ${ }^{[11-13]}$

Questions 5-9, 14, 18, and 21 were regarding the routinely recorded hemodynamic parameters and laboratory investigations common to all patients. SRs were significantly more aware than both the nurses and the JRs. However, the nurses had significantly better awareness as compared with the JRs for questions on TLC/DLC, electrolyte status, blood gas reports, intake/output, and any new organ failure. Although SRs order investigations in our ICU, yet role of nurses and JRs is also important as timely prompts from them might save redundant tests from being performed thus reducing medical costs. ${ }^{[14-18]}$ Nurses also have a definite role in surveillance of patients in any ICU in the form of pointing out early derangement of vital parameters and hence may decrease the mortality in ICUs. ${ }^{[19-21]}$

Questions pertaining to change of posture, pressure point changes, care of the tubes and catheters and physiotherapy of the patients (Q no. 10-13,15, 16, 24) were obligatory to the routine ICU care of the patients, and our analysis showed that the SRs were better than JRs. However, nurses were more aware than SRs for the question pertaining to perceptible pressure point changes. The incidence of pressure ulcers in ICU setup may range from $10 \%$ to $40 \%{ }^{[22-26]}$ Early risk assessment and mobilization are the strategies to reduce patient morbidity in those with expected longer duration of ICU stay. ${ }^{[27-33]}$ In our ICU, nurses provide general patient care in consort with JRs and any abnormality is reported to SRs. Disagreement in the awareness of the study groups may be suggestive of lack of adequate communication. Reader TW et al and Pronovost PJ et al. suggest a positive interaction between senior team members and the junior staff as being contributory to improved outcomes in ICUs. ${ }^{[34,35]}$ Team training programs boost communication and co-operative decision-making. ${ }^{[35]} \mathrm{An}$ example of this is seen through the analysis of responses to the question related to suctioning. All the three groups answered equally well as the protocol of our ICU dictates any two of the three group members to be present at all times during suctioning of the patients. Any change of the nature of the secretions has to be recorded by the team members, thereby minimizing errors.

Questions regarding weaning trial, DVT prophylaxis and inotropic support (Q no. 17, 19, 22) were aimed at evaluating the general treatment strategies in the ICU in which the SRs had better knowledge than the other groups. Interestingly, there 
was no significant difference between the knowledge of both the group of doctors for the question on weaning. Rose et al. suggested that greater involvement by the critical care nurses in decision-making regarding weaning strategies for patients may lead to better outcomes. ${ }^{[36]}$ In a large cross-sectional survey done by Rose et al., it was observed that despite variation in adoption of protocols related to ventilation and weaning across ICUs, a lack of collaboration between various professionals working as a team, might lead to delays in weaning and extubation. ${ }^{[37]}$ Nurses might be at an advantageous position, given the larger personnel to patient ratio to play an active role in the ventilatory management of patients. The question on feeding (Q no. 20) followed a sequence similar to earlier discussed questions. Early commencement of feeding of ICU patients (within 24-48 h of admission) as compared to late ( $>72 \mathrm{~h}$ after admission) has been shown to affect patient outcome and nurses have a role in providing clinical prompts to the doctors for the same. ${ }^{[38]}$ The question on DVT prophylaxis revealed a significantly better response from the SRs and nurses as compared to the trainee doctors. The importance of venous thromboembolism prophylaxis in an ICU is paramount, and ACCP has formulated guidelines and periodically revised the same for DVT prophylaxis ${ }^{[39]}$ Thus, from risk assessment to decision-making, the role of teamwork in the same is immense.

Questions 23 and 24 were related to taking informed consent in an ICU. There was no significant difference in the awareness for the same in all three study groups indicating proper communication between our teams. Informed consent for treatment and procedures being done is essential in any medical setup to avoid charges of assault and battery from the patients and their families and was therefore included in our questionnaire. ${ }^{40-42]}$

One limitation of the study was that this single-center data might be different from centers with a different structure of the ICU team. Another limitation was the filling of identical pro forma by the ICU team in which the doctors were rotated on a monthly basis whereas the nurses were stationary over the study period. Although theoretically this fact might lead to some degree of increased preparedness on the part of the ICU team members to answer the questionnaire subsequently for a different patient, yet no change in correct response rate to the questionnaire in any group was seen over the study period. This theory, although not backed up by evidence, could be remedied by spreading the study over a longer duration of time and not repeating the questionnaire to a single study group member. Consent for nondisclosure of questions could be taken from all subjects to prevent this.

\section{Conclusions}

Al though we did not find any significant difference between the knowledge of doctors and nurses, a subgroup analysis showed that among doctors SRs were far more aware of patient-related parameters than the other two groups. Thus to conclude, the main group in ICU on which the principal decision-making burden rests may be better aware than the others. The burden on the main decision maker may be relaxed by enthusiastic performance by other groups to aid the major decision-making process. This may lead to better patient care and outcomes in ICU. Thus regular audits in any health-care facility are vital for discovering loopholes in the management of the patient. We feel that Increasing education of the ICU team members toward explaining the relevance of patient-related parameters in ICU, empowering the nurses and JR doctors in the decision-making processes related to patient management to improve the interprofessional communication and timely decision-making thereby obviating many critical events in ICU, question-answer sessions during the grand rounds, weekly classes and case presentations to increase knowledge, communication and adherence to protocols and positive reinforcement by means of incentives such as extolling the efforts of ICU team members as best SR, JR, nurse over a 6-month period, on the ICU bulletin board along with rewarding their efforts at an institutional level through certificates of appreciation may help us achieve these ends.

Although these interventions might improve the cooperation amongst the ICU team members, yet these require further studies in terms of being helpful in bringing out changes in the awareness of patient-related parameters among them.

\section{Financial support and sponsorship}

Dr. RML Hospital and PGIMER, New Delhi.

\section{Conflicts of interest}

There are no conflicts of interest.

\section{RefERENCES}

1. Ferreira FL, Bota DP, Bross A, Mélot C, Vincent JL. Serial evaluation of the SOFA score to predict outcome in critically ill patients. JAMA 2001;286:1754-8.

2. Vincent JL, de Mendonça A, Cantraine F, Moreno R, Takala J, Suter PM, et al. Use of the SOFA score to assess the incidence of organ dysfunction/failure in Intensive Care Units: Results of a multicenter, prospective study. Working group on "sepsis-related problems" of the European Society of Intensive Care Medicine. Crit Care Med 1998;26:1793-800.

3. Moreno R, Vincent JL, Matos R, Mendonça A, Cantraine F, Thijs L, et al. The use of maximum SOFA score to quantify organ dysfunction/failure in intensive care. Results of a prospective, multicentre study. Working Group on Sepsis Related Problems of the ESICM. Intensive Care Med 1999;25:686-96.

4. Haesler E, editor. National Pressure Ulcer Advisory Panel, European Pressure Ulcer Advisory Panel and Pan Pacific Pressure Injury Alliance. Prevention and Treatment of Pressure Ulcers: Quick Reference Guide. Perth, Australia: Cambridge Media; 2014.

5. MacIntyre NR, Cook DJ, Ely EW Jr., Epstein SK, Fink JB, Heffner JE, et al. Evidence-based guidelines for weaning and discontinuing ventilatory support: A collective task force facilitated by the American College of Chest Physicians; the American Association for Respiratory Care; and the American College of Critical Care Medicine. Chest 2001;120:375S-95S.

6. Hunter S. Determination of moral negligence in the context of the undermedication of pain by nurses. Nurs Ethics 2000;7:379-91.

7. Davis R, Magilvy JK. Quiet pride: The experience of chronic illness by rural older adults. J Nurs Scholarsh 2000;32:385-90.

8. Aragon D. Evaluation of nursing work effort and perceptions about blood glucose testing in tight glycemic control. Am J Crit Care 2006;15:370-7. 
9. Curtis JR, Cook DJ, Wall RJ, Angus DC, Bion J, Kacmarek R, et al. Intensive Care Unit quality improvement: A "how-to" guide for the interdisciplinary team. Crit Care Med 2006;34:211-8.

10. Guidelines for Intensive Care Unit admission, discharge, and triage. Task Force of the American College of Critical Care Medicine, Society of Critical Care Medicine. Crit Care Med 1999;27:633-8.

11. Edwards R, Drumright L, Kiernan M, Holmes A. Covering more territory to fight resistance: Considering nurses' role in antimicrobial stewardship. J Infect Prev 2011;12:6-10.

12. Castledine G. Prescribing is not the only issue for nurses and drugs. $\mathrm{Br}$ J Nurs 2006;15:837.

13. Jutel A, Menkes DB. Nurses' reported influence on the prescription and use of medication. Int Nurs Rev 2010;57:92-7.

14. Jacobs P, Noseworthy TW. National estimates of intensive care utilization and costs: Canada and the United States. Crit Care Med 1990;18:1282-6.

15. Halpern NA. Can the costs of critical care be controlled? Curr Opin Crit Care 2009;15:591-6.

16. Mullins PM, Goyal M, Pines JM. National growth in Intensive Care Unit admissions from emergency departments in the united states from 2002 to 2009. Acad Emerg Med 2013;20:479-86.

17. May TA, Clancy M, Critchfield J, Ebeling F, Enriquez A, Gallagher C, et al. Reducing unnecessary inpatient laboratory testing in a teaching hospital. Am J Clin Pathol 2006;126:200-6.

18. Thavendiranathan P, Bagai A, Ebidia A, Detsky AS, Choudhry NK. Do blood tests cause anemia in hospitalized patients? The effect of diagnostic phlebotomy on hemoglobin and hematocrit levels. J Gen Intern Med 2005;20:520-4.

19. Rogers AE, Dean GE, Hwang WT, Scott LD. Role of registered nurses in error prevention, discovery and correction. Qual Saf Health Care 2008; 17:117-21.

20. Goldhill DR, McNarry AF. Physiological abnormalities in early warning scores are related to mortality in adult inpatients. $\mathrm{Br} \mathrm{J}$ Anaesth 2004;92:882-4.

21. Naeem N, Montenegro H. Beyond the Intensive Care Unit: A review of interventions aimed at anticipating and preventing in-hospital cardiopulmonary arrest. Resuscitation 2005;67:13-23.

22. VanGilder C, Amlung S, Harrison P, Meyer S. Results of the 2008-2009 International Pressure Ulcer Prevalence Survey and a 3-year, acute care, unit-specific analysis. Ostomy Wound Manage 2009;55:39-45.

23. Campbell KE, Woodbury MG, Houghton PE. Implementation of best practice in the prevention of heel pressure ulcers in the acute orthopedic population. Int Wound J 2010;7:28-40.

24. Ozdemir H, Karadag A. Prevention of pressure ulcers: A descriptive study in 3 Intensive Care Units in Turkey. J Wound Ostomy Continence Nurs 2008;35:293-300.

25. Nijs N, Toppets A, Defloor T, Bernaerts K, Milisen K, Van Den Berghe G, et al. Incidence and risk factors for pressure ulcers in the Intensive Care Unit. J Clin Nurs 2009;18:1258-66.
26. Bours GJ, De Laat E, Halfens RJ, Lubbers M. Prevalence, risk factors and prevention of pressure ulcers in Dutch Intensive Care Units. Results of a cross-sectional survey. Intensive Care Med 2001;27:1599-605.

27. Jastremski CA. Pressure relief bedding to prevent pressure ulcer development in critical care. J Crit Care 2002;17:122-5.

28. Suriadi, Sanada H, Sugama J, Thigpen B, Subuh M. Development of a new risk assessment scale for predicting pressure ulcers in an Intensive Care Unit. Nurs Crit Care 2008;13:34-43.

29. Reilly EF, Karakousis GC, Schrag SP, Stawicki SP. Pressure ulcers in the Intensive Care Unit: The 'forgotten' enemy. OPUS 12 Sci 2007;1:17-30.

30. Compton F, Hoffmann F, Hortig T, Strauss M, Frey J, Zidek W, et al. Pressure ulcer predictors in ICU patients: Nursing skin assessment versus objective parameters. J Wound Care 2008;17:417-20, 422-4

31. Defloor T, Grypdonck MF. Validation of pressure ulcer risk assessment scales: A critique. J Adv Nurs 2004;48:613-21.

32. Griffiths P, Jull A. How good is the evidence for using risk assessment to prevent pressure ulcers? Nurs Times 2010;106:10-3.

33. Cooper KL. Evidence-based prevention of pressure ulcers in the Intensive Care Unit. Crit Care Nurse 2013;33:57-66.

34. Reader TW, Flin R, Cuthbertson BH. Communication skills and error in the Intensive Care Unit. Curr Opin Crit Care 2007;13:732-6.

35. Pronovost PJ, Thompson DA, Holzmueller CG, Lubomski LH, Dorman T, Dickman F, et al. Toward learning from patient safety reporting systems. J Crit Care 2006;21:305-15.

36. Rose L, Nelson S, Johnston L, Presneill JJ. Decisions made by critical care nurses during mechanical ventilation and weaning in an Australian Intensive Care Unit. Am J Crit Care 2007; 16:434-43.

37. Rose L, Blackwood B, Egerod I, Haugdahl HS, Hofhuis J, Isfort M, et al. Decisional responsibility for mechanical ventilation and weaning: An international survey. Crit Care 2011;15:R295.

38. Heyland DK, Dhaliwal R, Drover JW, Gramlich L, Dodek P, Canadian Critical Care Clinical Practice Guidelines Committee et al. Canadian clinical practice guidelines for nutrition support in mechanically ventilated, critically ill adult patients. JPEN J Parenter Enteral Nutr 2003;27:355-73.

39. Guyatt GH, Akl EA, Crowther M, Gutterman DD, Schuünemann HJ, American College of Chest Physicians Antithrombotic Therapy and Prevention of Thrombosis Panel, et al. Executive summary: Antithrombotic therapy and prevention of thrombosis, $9^{\text {th }}$ ed.: American college of chest physicians evidence-based clinical practice guidelines. Chest 2012;141:7S-47S.

40. Bille DA. The nurse's role in informed consent. QRB Qual Rev Bull 1980;6:25-9.

41. Nichita EC, Buckley PF. Informed consent and competency: Doctor's dilemma on the consultation liaison service. Psychiatry (Edgmont) 2007;4:53-5

42. Bowman D, Spicer J, Iqbal R. Informed Consent: A Primer for Clinical Practice. New York: Cambridge University Press; 2011. 